\title{
Validation and comparative assessment of low anterior resection syndrome questionnaires in Greek rectal cancer patients
}

\author{
Artemis Liapi ${ }^{a}$, Constantine Mavrantonis ${ }^{b}$, Panagiotis Lazaridis $^{b}$, Eleni Kourkounic, Andreas Zevlas ${ }^{a}$, \\ George Zografos ${ }^{d}$, George Theodoropoulos ${ }^{d}$ \\ "G. Gennimatas" General Hospital of Athens; "Hygeia" Hospital, Athens; Center for Clinical Epidemiology and \\ Outcomes Research (CLEO), Athens; Athens Medical School, Greece
}

\section{Abstract}

${ }^{a} 1^{\text {st }}$ Surgical Department, "G. Gennimatas" General Hospital of Athens (Artemis Liapi, Andreas Zevlas); ${ }^{b} 6^{\text {th }}$ Department of Surgery, "Hygeia" Hospital (Constantine Mavrantonis, Panagiotis Lazaridis); 'Center for Clinical Epidemiology and Outcomes Research (CLEO) (Eleni Kourkouni); ${ }^{\mathrm{d}} \mathrm{st}^{\text {st }}$ Department of Propaedeutic Surgery, Athens Medical School (George Zografos, George Theodoropoulos), Athens, Greece

Correspondence to: Artemis Liapi, 154 Mesogeion Ave., 11527 Athens, Greece, e-mail: artemis1504@hotmail.com

Conflict of Interest: None

Received 6 August 2018; accepted 2 November 2018; published online 15 January 2019

DOI: https://doi.org/10.20524/aog.2019.0350

\section{Introduction}

Mesorectal clearance and neoadjuvant radiotherapy have led to superior oncologic outcomes for rectal cancer patients $[1,2]$. Sphincter preservation techniques are applied to the vast majority of locally advanced rectal cancers [3]. Avoidance of permanent stoma is achieved at the cost of unavoidable functional disturbances, such as increased bowel frequency, urgency, fecal incontinence, and evacuation difficulty, collectively referred to as low anterior resection syndrome (LARS) $[4,5]$.

The heterogeneity of symptoms and the lack of validated systems to capture the LARS have led to inadequate 
assessment of this syndrome's impact, which may persist for years [4]. Initial attempts at symptom scoring were mainly focused on fecal incontinence instruments and were not able to reveal the complexity of LARS [6-9]. In 2005, the colorectal functional outcome (COREFO) questionnaire, as a modification of the existing Vaizey scale, was introduced as a more thorough, self-completing instrument [10]. In the same year, the Memorial Sloan-Kettering Cancer Center bowel function instrument (MSKCC-BFI) was also introduced [11]. In 2012, an accurate, brief questionnaire, the LARS score, was developed, containing the 5 most bothersome LARS symptoms [12]. The LARS score was then extensively validated in several countries [13-15]. Bowel function-related quality of life (QoL) instruments, such as the European Organisation for Research and Treatment of Cancer's Quality of Life Questionnaire (EORTC QLQ-C30), with the addition of the Colorectal Module QLQ-CR29, have been used complementarily to evaluate LARS and also to validate rectal dysfunction questionnaires $[17,18]$.

Our goal was to assess the LARS score, COREFO and MSKCC-BFI questionnaires simultaneously in parallel with EORTC QLQ-C30 and QLQ-CR29 questionnaires in a series of patients after sphincter-preserving surgery for rectal cancer, in order to validate all 3 questionnaires in Greece. An additional objective was to conduct a comparative study of the 3 questionnaires with the purpose of revealing the most convenient one for the assessment of LARS.

\section{Patients and methods}

\section{Study population}

From the 325 rectal cancer patients' medical records initially reviewed, 112 patients were deemed eligible and eventually entered the study. Included patients had been treated in either an academic unit, a National Health System hospital or a private institution during the period 2007-2017. Inclusion criteria were: rectal adenocarcinoma up to $15 \mathrm{~cm}$ from the anal verge, low anterior resection (LAR) with either partial or total mesorectal excision (PME or TME), with or without intersphincteric resection and with or without defunctioning protective ileostomy, in the latter case with the stoma reversed at least 6 months prior to assessment. Exclusion criteria included recurrence, metastatic disease, presence of stoma, dementia, and inability to speak Greek. The sample description is shown in Table 1 and the flowchart of the study in Fig. 1. Informed consent was obtained from every patient who participated in the study. The study was approved by the Medical Research Ethics Committee of each hospital and was conducted in accordance with the Declaration of Helsinki (1989) of the World Medical Association. During their visit to a dedicated colorectal cancer clinic, patients were either interviewed personally or asked to answer the questionnaires by themselves, with or without assistance from a family member.
Table 1 Sample description (N=112 patients)

\begin{tabular}{lc}
\hline Characteristics & $\mathrm{N}(\%)$ \\
\hline Sex & \\
Female & $40(35.7 \%)$ \\
Male & $72(64.3 \%)$ \\
Age (years) & \\
$<70$ & $62(55.4 \%)$ \\
$\geq 70$ & $50(44.6 \%)$ \\
Tumor distance & \\
$\leq 10$ cm & $74(66.1 \%)$ \\
$>10$ cm & $38(33.9 \%)$ \\
Temporary stoma & \\
No & $43(38.4 \%)$ \\
Yes & $69(61.6 \%)$ \\
Stage & \\
I & $37(33.0 \%)$ \\
II & $30(26.8 \%)$ \\
III & $45(40.2 \%)$ \\
(Neo)adjuvant radiotherapy & \\
No & $63(56.2 \%)$ \\
Yes & $49(43.8 \%)$ \\
Open/Laparoscopic & $20(17.9 \%)$ \\
Open & $47(42.0 \%)$ \\
\hline Laparoscopic & \\
$>12$ & \\
\hline & \\
\hline & \\
\hline & \\
\hline & \\
\hline
\end{tabular}

\section{Translation}

Greek versions of EORTC QLQ-C30 and QLQ-CR29 questionnaires were used. After permission had been obtained from the original authors, the English versions of the 3 bowel dysfunction questionnaires were translated into Greek using a forward and back translation process, according to the current recommendations from the World Health Organization (WHO) and the EORTC [19,20]. After translation, questionnaires were given to selected patients to determine if the questions appeared clear, understandable and in logical order (face validity).

\section{Test-retest reliability}

Repeat questionnaires were given to a number of patients 7-14 days after the initial answers in order to assess their testretest reliability. 


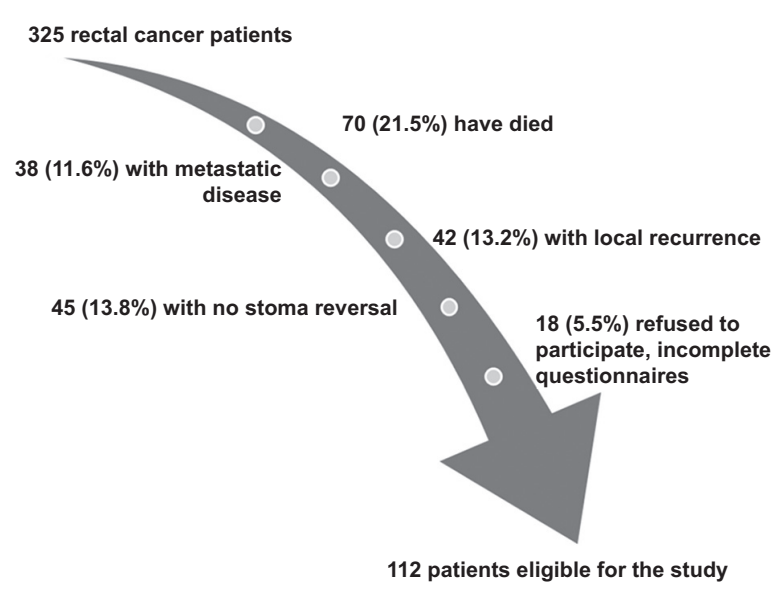

Figure 1 Flowchart of the study

\section{LARS score questionnaire}

The most important QoL-affecting LARS score questionnaire items are: incontinence to flatus, incontinence to liquid stools, frequency, clustering and urgency. Highest scores are given to clustering and urgency. The LARS score consists of 5 questions and total scores range from 0-42. A score of 0-20 indicates no LARS, a score of 21-29 minor LARS, and a score of 30-42 major LARS [12].

\section{COREFO questionnaire}

The questionnaire consists of 27 questions and has 5 subscales (incontinence, social impact, frequency, stool aspects, need for medication). The responses are given on a 5-point Likert scale for all items, apart from the 2 that ask the frequency of bowel movements. Higher scores indicate worse bowel function [10].

\section{MSKCC-BFI questionnaire}

The MSKCC-BFI questionnaire includes 18 items involving 3 subscales, 4 individual items of clinical significance and one total score that is obtained by summing all 18 items [11]. The responses are given on a 5-point Likert scale for all items, apart from the item that asks the frequency of bowel movements. Higher scores indicate better bowel function.

\section{EORTC QLQ-C30 and QLQ-CR29 questionnaires}

The EORTC QLQ-C30 comprises 30 questions. Five functional scales, 3 symptom scales, a global health status/ QoL scale, and 6 single items are included. Functional severity scales range from 0 (worst function) to 100 (best function). The symptom severity scales range from 0 (least severe) to 100 (most severe) $[17,21]$. The EORTC QLQ-CR29 is an updated module based on the EORTC QLQ-CR38 questionnaire; it is a colorectal-specific QoL questionnaire and includes 4 functional subscales and several subscales [18].

\section{Statistical analysis}

Internal consistency for the subscales of questionnaires was assessed using Cronbach's alpha coefficient [22]. The range of index values is from $0-1$. Large values suggest broad consistency of the questions that comprise the subscale. The repeatability test (test-retest) was performed by applying the statistical intraclass correlation coefficient (ICC) criterion. This criterion takes values between -1 and +1 . Values close to 1 indicate a high repeatability of the questionnaire. Test-retest reliability was also evaluated using a BlandAltman plot with 95\% limits of agreement (not shown in results). Results for repeatability are presented with ICC and $95 \%$ confidence intervals. Construct validity and associations between questionnaires' subscales (convergent validity) were evaluated with Spearman's correlation (rho). The convergent validity was determined by computing the correlations between each of the 3 questionnaires (LARS score, MSKCC-BFI, COREFO) and the EORTC QLQ-C30 and QLQ-CR29. Discriminant validity was investigated by the ability of the 3 questionnaires to differentiate between groups of patients with different clinical features, such as age, sex, postoperative period, temporary stoma, tumor distance from the anal verge, and neoadjuvant radiation therapy. Categorical data were expressed as absolute and relative (\%) frequencies, and continuous data as either mean and standard deviation, or median and interquartile range. Since the data were not normally distributed (tested by Kolmogorov-Smirnov and graphically with Q-Q plots) Mann-Whitney and Kruskal-Wallis tests were used to evaluate the association between scales and patient characteristics (discriminant validity). The level of statistical significance was set to alpha $5 \%$. All analyses were conducted using SPSS v22 (SPSS Inc., Chicago, IL).

\section{Results}

\section{Internal consistency and repeatability}

Table 2 presents the results of the internal consistency and test-retest checks for each subscale of the questionnaires. The internal consistency check was performed on the subscales where it was applicable. Overall, the internal consistency of the subscales was satisfactory (Cronbach's a $>0.6$ ), apart from the stool aspects subscale of the COREFO questionnaire, where the internal consistency was moderate (Cronbach's $\mathrm{a}=0.533$ ). The repeatability test showed that all the subscales were characterized by extremely high reproducibility (ICC $>0.9)$. 
Table 2 Internal consistency and repeatability of questionnaires

\begin{tabular}{lccr}
\hline Variable & Number of questions & Cronbach's a & ICC (95\%CI) \\
\hline LARS score & 5 & $-^{*}$ & $0.956(0.873-0.985)$ \\
\hline & COREFO questionnaire & & $0.998(0.994-0.999)$ \\
\hline Incontinence & 9 & 0.867 & $0.992(0.977-0.997$ \\
Social impact & 9 & 0.813 & $0.985(0.957-0.995)$ \\
Frequency & 2 & $-*$ & $0.966(0.901-0.988)$ \\
Stool aspects & 3 & 0.533 & $0.992(0.977-.0997)$ \\
Need for medication & 3 & 0.719 & $0.998(0.993-0.999)$ \\
\hline Total Score & 26 & - & \\
\hline
\end{tabular}

MSKCC-BFI questionnaire

\begin{tabular}{lccc}
\hline Frequency & 6 & 0.596 & $0.967(0.904-0.989)$ \\
Diet & 4 & 0.605 & $0.992(0.978-0.997)$ \\
Urgency & 4 & 0.874 & $0.994(0.983-0.998)$ \\
Incomplete evacuation & 1 & $-* *$ & $0.982(0.946-.0994)$ \\
Clustering & 1 & $-* *$ & $0.977(0.933-0.992)$ \\
Difference between gas/bowel movement & 1 & $-* *$ & $0.915(0.766-0.971)$ \\
Incontinence of flatus & 1 & $-* *$ & $0.999(0.998-0.999)$ \\
Global score & 10 & 0.662 & $0.964(0.896-0.988)$ \\
\hline Total score & 18 & 0.841 & $0.951(0.861-0.983)$ \\
\hline
\end{tabular}

${ }^{*}$ not calculated because sub-questions are differently scored

${ }^{*}$ not calculated because there is only one sub-question

ICC, intraclass correlation coefficient; LARS, low anterior resection syndrome; COREFO, colorectal functional outcome questionnaire; MSKCC-BFI, Memorial Sloan-Kettering Cancer Center bowel function instrument; 95\%CI, 95\% confidence interval

\section{Construct validity}

\section{LARS construct validity}

Highly positive correlations $(\mathrm{P}<0.001)$ were detected between LARS total score and each of the questions. Specifically, total score was significantly interrelated with incontinence to flatus (question $1, \mathrm{rho}=0.558$ ), incontinence to liquid stool (question 2, rho=0.550), bowel frequency (question 3, rho=0.548), clustering (question 4, rho=0.682) and urgency (question 5, rho=0.898). The fact that all questions were so strongly associated with the total LARS score indicates that this is a well and carefully structured valid questionnaire.

\section{COREFO construct validity}

Total COREFO score was highly positively correlated with all subscale scores (rho>0.4). In addition, all COREFO subscale scores were correlated with each other (rho>0.4), except for the stool-aspects subscale, which was not adequately correlated with either the incontinence or the frequency subscale $($ rho $<0.2)$. Overall, the COREFO questionnaire had valid construction.

\section{MSKCC-BFI construct validity}

Total MSKCC-BFI score was highly correlated with all subscale scores (rho>0.5), indicating the valid construction of the questionnaire. In addition, a high positive correlation was detected between many subscales (rho $>0.3$ ) (analytical data not shown).

\section{Convergent validity}

\section{LARS convergent validity}

The LARS questionnaire demonstrated good convergent validity through significant correlations with comparable domains of the EORTC QLQ-C30 and QLQ-CR29 questionnaires (Table 3). A highly negative correlation was detected between LARS total score and the QoL subscale of the EORTC QLQ-C30 questionnaire ( $\mathrm{rho}=-0.628$ ). Thus, the higher a patient's LARS score, the lower the expected QoL. Likewise, statistically significant negative correlations were detected between LARS and functional scales $(\mathrm{rho}<-0.3)$. On the other hand, the LARS score was significantly positively correlated with fatigue and diarrhea subscales ( $\mathrm{rho}=0.443$ and rho $=0.556$, respectively). The LARS score was also 
Table 3 Convergent validity of the low anterior resection syndrome (LARS) score with the European Organisation for Research and Treatment of Cancer's (EORTC) QLQ-C30 and QLQ-CR29 qualityof-life (QoL) questionnaires. Only statistically significant correlations are demonstrated

\begin{tabular}{llc}
\hline Variable & \multicolumn{2}{c}{ LARS score } \\
\cline { 2 - 3 } & Spearman's rho & P-value \\
\hline
\end{tabular}

\begin{tabular}{lcc}
\hline & EORTC QLQ-C30 & \\
\hline QoL & -0.628 & $<0.001$ \\
Physical functioning & -0.270 & 0.007 \\
\hline Role functioning & -0.609 & $<0.001$ \\
Emotional functioning & -0.332 & 0.001 \\
\hline Social functioning & -0.519 & $<0.001$ \\
Fatigue & 0.443 & $<0.001$ \\
\hline Nausea and vomiting & 0.254 & 0.011 \\
Pain & 0.235 & 0.019 \\
\hline Dyspnea & 0.295 & 0.003 \\
\hline Insomnia & 0.205 & 0.042 \\
\hline Diarrhea & 0.556 & $<0.001$ \\
Financial difficulties & 0.188 & 0.063 \\
\hline
\end{tabular}

EORTC QLQ-CR29

\begin{tabular}{lcc}
\hline Anxiety & 0.292 & 0.003 \\
Body image & 0.348 & $<0.001$ \\
\hline Abdominal pain & 0.244 & 0.015 \\
Defecation problems & 0.572 & $<0.001$ \\
\hline Incontinence & 0.707 & $<0.001$ \\
Bloated feeling & 0.250 & 0.013 \\
\hline Sore skin & 0.294 & 0.003 \\
\hline Embarrassment & 0.610 & $<0.001$ \\
\hline
\end{tabular}

positively correlated with important EORTC QLQ-CR29 domains (Table 3).

\section{COREFO convergent validity}

The COREFO questionnaire demonstrated good convergent validity through significant correlations with comparable domains of the EORTC QLQ-C30 and CR29 questionnaires (analytical data not shown). A high negative correlation was detected between COREFO total score and the QoL subscale of the EORTC QLQ-C30 questionnaire (rho=-0.703). Likewise, statistically significant negative correlations were detected between COREFO total score and role functioning, emotional functioning and social functioning $(r h o<-0.3)$. On the other hand, COREFO total score was significantly positively correlated with fatigue, pain and diarrhea subscales (rho $=0.385$, rho $=0.304$ and $\mathrm{rho}=0.563$, respectively). With regard to EORTC QLQ-CR29, COREFO total score was significantly positively correlated with anxiety, body image, abdominal pain, defecation problems, incontinence, sore skin and embarrassment (rho $>0.3$ ).

\section{MSKCC-BFI convergent validity}

A statistically significant positive correlation was detected between MSKCC-BFI total score and the QoL subscale of the EORTC QLQ-C30 questionnaire (rho=0.641, analytical data not shown). Positive correlations were also detected between MSKCC-BFI total score and role functioning, emotional functioning and social functioning ( $r h o=0.569$, rho $=0.389$ and rho $=0.517$, respectively). On the other hand, MSKCC-BFI total score was significantly negatively correlated with the fatigue and diarrhea subscales (rho $=-0.379$ and $\mathrm{rho}=-0.634$ respectively). As regards EORTC QLQ-CR29, MSKCC-BFI total score was significantly negatively correlated with body image, defecation problems, incontinence, sore skin and embarrassment (rho<0.3 , analytical data not shown).

\section{Discriminant validity}

Statistically significant associations were detected between LARS, COREFO, MSKCC-BFI scores and tumor distance, as well as temporary stoma $(\mathrm{P}<0.001$ and $\mathrm{P}=0.009$, $\mathrm{P}<0.001$ and $\mathrm{P}=0.005, \mathrm{P}<0.001$ and $\mathrm{P}=0.002$ respectively, Table 4). In addition, COREFO and MSKCC-BFI scores were significantly associated with neoadjuvant radiation therapy. Patients with tumor distance $\leq 10 \mathrm{~cm}$ and those who had a temporary stoma had higher LARS and COREFO scores but lower MSKCC-BFI score than those with tumor distance $>10 \mathrm{~cm}$ and those with no stoma. Likewise, patients who had undergone radiotherapy had higher COREFO score and lower MSKCC-BFI (Table 4).

\section{Associations between questionnaires}

LARS total score was significantly positively correlated with all the subscales of the COREFO questionnaire, as well as the COREFO total score, and especially with incontinence and social impact (rho 0.703 and rho 0.861) (Table 5). Statistically significant negative correlations were detected between LARS score and all MSKCC-BFI subscales, notably urgency and clustering (rho -0.785 and rho -0.666) (Table 5). Lastly, COREFO incontinence and social impact scales, as well as COREFO total score, were significantly negatively correlated with all MSKCC-BFI subscales (analytical data not shown). COREFO frequency subscale was positively correlated with MSKCC-BFI frequency subscale $(\mathrm{rho}=0.317)$ and negatively correlated with diet, urgency, incomplete evacuation and clustering on the MSKCC-BFI questionnaire. Stool aspects of the COREFO questionnaire were negatively correlated with incomplete evacuation and total score on the MSKCCBFI (rho $=-0.420$ and $r h o=-0.325$, respectively). The need for 
Table 4 Discriminant validity of LARS, COREFO and MSKCC-BFI scores

\begin{tabular}{|c|c|c|c|c|c|c|}
\hline Characteristics & Median (IQR) & P-value & Median (IQR) & P-value & Median (IQR) & $\mathrm{P}$-value \\
\hline Sex & & 0.754 & & 0.434 & & 0.889 \\
\hline Female & $30(20-35)$ & & $42(36-60)$ & & $71(62-79)$ & \\
\hline Male & $26(15-38)$ & & $47(36-65)$ & & $70(62-80)$ & \\
\hline Age (years) & & 0.189 & & 0.677 & & 0.528 \\
\hline$<70$ & $31(18-38)$ & & $46(37-61)$ & & $70(62-80)$ & \\
\hline$\geq 70$ & $24(16-32)$ & & $45(36-63)$ & & $70(58-78)$ & \\
\hline Tumor distance & & $<0.001$ & & $<0.001$ & & $<0.001$ \\
\hline$\leq 10 \mathrm{~cm}$ & $32(23-39)$ & & $53(42-65)$ & & $67(59-75)$ & \\
\hline$>10 \mathrm{~cm}$ & $15(9-22)$ & & $35(31-40)$ & & $80(69-83)$ & \\
\hline Temporary stoma & & 0.009 & & 0.005 & & 0.002 \\
\hline No & $22(9-32)$ & & $38(32-58)$ & & $76(68-83)$ & \\
\hline Yes & $30(20-39)$ & & $51(41-65)$ & & $67(60-75)$ & \\
\hline Stage & & 0.430 & & 0.624 & & 0.709 \\
\hline I & $26(16-34)$ & & $42(37-57)$ & & $72(63-78)$ & \\
\hline II & $23(13-38)$ & & $49(32-63)$ & & $70(63-80)$ & \\
\hline III & $32(20-38)$ & & $48(37-64)$ & & $69(58-79)$ & \\
\hline (Neo)adjuvant radiotherapy & & 0.072 & & 0.030 & & 0.014 \\
\hline No & $25(16-35)$ & & $42(35-61)$ & & $72(63-80)$ & \\
\hline Yes & $33(21-41)$ & & $58(45-66)$ & & $63(58-72)$ & \\
\hline Open/Laparoscopic & & 0.997 & & 0.475 & & 0.558 \\
\hline Open & $28(19-36)$ & & $44(36-60)$ & & $69(62-80)$ & \\
\hline Laparoscopic & $26(16-38)$ & & $48(37-64)$ & & $71(60-78)$ & \\
\hline $\begin{array}{l}\text { Length of } \\
\text { postoperative period (months) }\end{array}$ & & 0.530 & & 0.733 & & 0.286 \\
\hline$\leq 12$ & $31(16-38)$ & & $48(37-63)$ & & $71(62-80)$ & \\
\hline$>12$ & $25(16-35)$ & & $42(36-61)$ & & $69(60-76)$ & \\
\hline
\end{tabular}

LARS, low anterior resection syndrome; COREFO, colorectal functional outcome questionnaire; MSKCC-BFI, Memorial Sloan-Kettering Cancer Center bowel function instrument; $I Q R$, interquartile range

medication COREFO subscale was negatively correlated with all MSKCC-BFI subscales (analytical data not shown).

\section{Discussion}

The present study achieved validation of Greek versions of the LARS score, COREFO and MSKCC-BFI questionnaires in rectal cancer patients. All 3 questionnaires were validated through the same validation process and all demonstrated convincing psychometric properties regarding internal consistency (Cronbach's a $>0.6$ ), repeatability (ICC>0.9) and construct validity (rho>0.5). Specifically for the LARS score, our findings are consistent with previous validation attempts and existing international versions [13-16].

Other LARS score validations used a single QoL question to investigate convergent validity, rather than the 2 instruments of EORTC used in our study $[13,14,16]$. The latter may not have provided the opportunity to evaluate specific QoL subscales. Similarly to the Chinese validation process, we investigated the convergent validity of LARS by analyzing its correlation with the rather extensive EORTC QoL instruments [15]. LARS, COREFO and MSKCC-BFI scores were significantly associated with tumor distance as well as temporary stoma. Low-lying tumors and temporary stoma construction appear to be associated with bowel dysfunction severity, as in other countries' LARS score versions [16,15]. In contrast to COREFO and MSKCC-BFI scores, LARS score did not indicate that radiotherapy was an aggravating factor for postoperative bowel dysfunction. In line with the Lithuanian validation study, the lack of such an association may be explained by the rather smaller percentage of our patients' sample that had been preoperatively irradiated [14]. According to other validation results, patients have significantly higher LARS scores after neoadjuvant radiotherapy [13].

Apart from anatomical and functional alterations, anastomotic level, age, sex, surgeon, stage, type of surgery 
Table 5 Correlations between LARS and COREFO, MSKCC-BFI questionnaires

\begin{tabular}{llc}
\hline Variable & \multicolumn{2}{c}{ LARS score } \\
\cline { 2 - 3 } & rho & $\mathrm{P}$ \\
\hline
\end{tabular}

\begin{tabular}{lll}
\hline \multicolumn{3}{c}{ COREFO } \\
\hline Incontinence & 0.703 & $<0.001$ \\
Social impact & 0.861 & $<0.001$ \\
Frequency & 0.406 & $<0.001$ \\
Stool aspects & 0.371 & $<0.001$ \\
Need for medication & 0.567 & $<0.001$ \\
Total & 0.864 & $<0.001$ \\
\hline
\end{tabular}

MSKCC-BFI

\begin{tabular}{lll}
\hline Frequency & -0.450 & $<0.001$ \\
\hline Diet & -0.426 & $<0.001$ \\
\hline Urgency & -0.785 & $<0.001$ \\
\hline Incomplete evacuation & -0.601 & $<0.001$ \\
\hline Clustering & -0.666 & $<0.001$ \\
\hline Different between gas/bowel movement & -0.535 & $<0.001$ \\
\hline Incontinence of flatus & -0.605 & $<0.001$ \\
\hline Global score & -0.569 & $<0.001$ \\
\hline Total score & -0.763 & $<0.001$ \\
\hline
\end{tabular}

LARS, low anterior resection syndrome; COREFO, colorectal functional outcome questionnaire; MSKCC-BFI, Memorial Sloan-Kettering Cancer Center bowel function instrument

(open/laparoscopic), length of postoperative period, temporary diversion ileostomy, adjuvant treatments, septic complications, and the use of a reservoir, have all been proposed as risk factors for LARS [23-26]. It is imperative to clarify such predisposing factors so that it is possible to recognize patients at high risk of suffering LARS. The value of suggested intraoperative (i.e., colonic J-pouch) and postoperative interventions (biofeedback, sacral neuromodulation) for LARS prevention and management can then be further clarified [27-29]. A common symptom-based scoring system for bowel dysfunction after LAR for rectal cancer is undoubtedly of value. This will help in functional subgrouping for clinical interventions under investigation for LARS, as well as in scientific communication, by using a common language when analyzing postoperative bowel dysfunction. Validation of international instruments assessing LARS is of key importance.

The international literature lacks evidence for the type of comparative assessment we performed in the current study. All 3 questionnaires were given to the same population and at the same time. The conclusions from the comparative study among the 3 questionnaires are both direct, through correlations of the overall scores and their subscales, and indirect, through correlations with the EORTC questionnaires. As expected, multiple correlations were observed between the most extensive and analytical questionnaires (COREFO and
MSKCC-BFI) and the various EORTC QLQ-C30 and CR29 subscales. Nevertheless, it was notable that the task-oriented anorectal function questions of the short and quick to complete LARS questionnaire achieved meaningful correlations with significant functionality subdomains.

The use of one questionnaire as an instrument to detect LARS does not preclude the use of another, depending on the purposes of the study being carried out. All 3 questionnaires have the ability to reveal patients who experience LARS. The COREFO and MSKCC-BFI questionnaires seem to be more suitable for comprehensive and in-depth evaluation of LARS. The LARS score, having only 5 targeted questions and without missing the core LARS symptomatology, may be considered as the most appropriate, rapid and accurate tool for screening and prospectively reassessing LARS.

\section{Summary Box}

\section{What is already known:}

- There is a lack of validated systems to evaluate low anterior resection syndrome (LARS) in Greece

- The LARS score has been extensively validated and has been translated from the original Danish version into several languages

- There is no previous comparative study of LARS questionnaires in Greek rectal cancer patients

\section{What the new findings are:}

- This was the first study to validate the LARS score questionnaire, colorectal functional outcome questionnaire (COREFO) and Memorial SloanKettering Cancer Center bowel function instrument (MSKCC-BFI) in Greek rectal cancer patients

- The Greek versions of the LARS score, COREFO and MSKCC-BFI questionnaires were proven to have good psychometric properties and can be used as specific and valid instruments for measuring LARS

- Comparative assessment of the 3 questionnaires revealed that LARS score may be established as the simplest and fastest to complete targeted tool for assessing LARS

\section{References}

1. Sebag-Montefiore D, Stephens RJ, Steele R, et al. Preoperative radiotherapy versus selective postoperative chemoradiotherapy in patients with rectal cancer (MRC CR07 and NCIC-CTG C016): a multicentre, randomised trial. Lancet 2009;373:811-820.

2. van Gijn W, Marijnen CA, Nagtegaal ID, et al; Dutch Colorectal Cancer Group. Preoperative radiotherapy combined with total mesorectal excision for resectable rectal cancer: 12-year follow-up of the multicentre, randomised controlled TME trial. Lancet Oncol 2011;12:575-582. 
3. Enker WE. Total mesorectal excision-the new golden standard of surgery for rectal cancer. Ann Med 1997;29:127-133.

4. Bryant CL, Lunniss PJ, Knowles CH, Thaha MA, Chan CL. Anterior resection syndrome. Lancet Oncol 2012;13:e403-e408.

5. Camilleri-Brennan J, Steele RJ. Quality of life after treatment for rectal cancer. Br J Surg 1998;85:1036-1043.

6. Jorge JM, Wexner SD. Etiology and management of fecal incontinence. Dis Colon Rectum 1993;36:77-97.

7. Vaizey CJ, Carapeti E, Cahill JA, Kamm MA. Prospective comparison of faecal incontinence grading systems. Gut 1999;44:77-80.

8. Rockwood TH, Church JM, Fleshman JW, et al. Patient and surgeon ranking of the severity of symptoms associated with fecal incontinence. Dis Colon Rectum 1999;42:1525-1532.

9. Chen TY-T, Emmertsen KJ, Laurberg S. What are the best questionnaires to capture anorectal function after surgery in rectal cancer? Curr Colorectal Cancer Rep 2015;11:37-43.

10. Bakx R, Sprangers MA, Oort FJ, et al. Development and validation of a colorectal functional outcome questionnaire. Int $J$ Colorectal Dis 2005;20:126-136.

11. Temple LK, Bacik J, Savatta SG, et al. The development of a validated instrument to evaluate bowel function after sphincter-preserving surgery for rectal cancer. Dis Colon Rectum 2005;48:1353-1365.

12. Emmertsen KJ, Laurberg S. Low anterior resection syndrome score: development and validation of a symptom-based scoring system for bowel dysfunction after low anterior resection for rectal cancer. Ann Surg 2002;2555:922-928.

13. Juul $\mathrm{T}$, Ahlberg $\mathrm{M}$, Biondo $\mathrm{S}$, et al. International validation of the low anterior resection syndrome score. Ann Surg 2014;259:728-734.

14. Samalavicius NE, Dulskas A, Lasinskas M, Smailyte G. Validity and reliability of a Lithuanian version of low anterior resection syndrome score. Tech Coloproctol 2016;20:215-220.

15. Hou XT, Pang D, Lu Q, et al. Validation of the Chinese version of the low anterior resection syndrome score for measuring bowel dysfunction after sphincter-preserving surgery among rectal cancer patients. Eur J Oncol Nurs 2015;19:495-501.

16. Juul T, Battersby NJ, Christensen P, et al; UK LARS Study Group. Validation of the English translation of the low anterior resection syndrome score. Colorectal Dis 2015;17:908-916.

17. Aaronson NK, Ahmedzai S, Bergman B, et al. The European Organization for Research and Treatment of Cancer QLQ-C30: a quality-of-life instrument for use in international clinical trials in oncology. J Natl Cancer Inst 1993;85:365-376.
18. Whistance RN, Conroy T, Chie W, et al; European Organisation for the Research and Treatment of Cancer Quality of Life Group. Clinical and psychometric validation of the EORTC QLQ-CR29 questionnaire module to assess health-related quality of life in patients with colorectal cancer. Eur J Cancer 2009;45:3017-3026.

19. World Health Organization. Process of translation and adaptation of instruments. 2010. http://www.who.int/substance_abuse/ research_tools/translation/en/. [Accessed 29 November, 2018]

20. Dewolf L, Koller M, Velikova G, Johnson C, Scott N, Bottomley A, EORTC Quality of Life Group. EORTC quality of life group translation procedure. Brussels 2009. https://www.eortc.org/app/ uploads/sites/2/2018/02/SCmanual.pdf. [Accessed 2 December, 2018]

21. Fayers P, Aaronson N. The EORTC QLQ-C30 Scoring Manual, $3^{\text {rd }}$ edition. Brussels, Belgium: EORTC Data Center, 2001.

22. Cronbach LJ. Coefficient alpha and the internal structure of tests. Psychometrika 1951;16:297-334.

23. Theodoropoulos GE, Papanikolaou IG, Karantanos T, Zografos G. Post-colectomy assessment of gastrointestinal function: a prospective study on colorectal cancer patients. Tech Coloproctol 2013;17:525-536.

24. Hughes DL, Cornish J, Morris C; LARRIS Trial Management Group. Functional outcome following rectal surgery-predisposing factors for low anterior resection syndrome. Int $J$ Colorectal Dis 2017;32:691-697.

25. Carrillo A, Enríquez-Navascués JM, Rodríguez A, et al. Incidence and characterization of the anterior resection syndrome through the use of the LARS scale (low anterior resection score). Cir Esp 2016;94:137-143.

26. Wells CI, Vather R, Chu MJ, Robertson JP, Bissett IP. Anterior resection syndrome-a risk factor analysis. J Gastrointest Surg 2015; 19:350-359.

27. Ziv Y, Zbar A, Bar-Shavit Y, Igov I. Low anterior resection syndrome (LARS): cause and effect and reconstructive considerations. Tech Coloproctol 2013;17:151-162.

28. Visser WS, Te Riele WW, Boerma D, van Ramshorst B, van Westreenen HL. Pelvic floor rehabilitation to improve functional outcome after a low anterior resection: a systematic review. Ann Coloproctol 2014;30:109-114.

29. Ramage L, Qiu S, Kontovounisios C, Tekkis P, Rasheed S, Tan E. A systematic review of sacral nerve stimulation for low anterior resection syndrome. Colorectal Dis 2015;17:762-771. 